\title{
Weighting and Pruning of Decision Rules by Attributes and Attribute Rankings
}

\author{
Urszula Stańczyk ${ }^{(\bowtie)}$ \\ Silesian University of Technology, Akademicka 16, 44-100 Gliwice, Poland \\ urszula.stanczyk@polsl.pl
}

\begin{abstract}
Pruning is a popular post-processing mechanism used in search for optimal solutions when there is insufficient domain knowledge to either limit learning data or govern induction in order to infer only the most interesting or important decision rules. Filtering of generated rules can be driven by various parameters, for example explicit rule characteristics. The paper presents research on pruning rule sets by two approaches involving attribute rankings, the first relaying on selection of rules referring to the highest ranking attributes, which is compared to weighting of rules by calculated quality measures dependent on weights coming from attribute rankings that results in rule ranking.
\end{abstract}

Keywords: Decision rules $\cdot$ Pruning $\cdot$ Weighting $\cdot$ Attribute $\cdot$ Ranking

\section{Introduction}

Rule classifiers express patterns discovered in data in learning processes through conditions on attributes included in the premises and pointing to specific classes [5]. A variety of available approaches to induction enable construction of classifiers with minimal numbers of constituent rules, with all rules that can be inferred from the training samples, or with subsets of interesting elements [3].

To limit the number of considered rules [9] either pre-processing can be employed, with reducing rather data than rules, by selection of features or instances, or in-processing relaying on induction of only those rules that satisfy given requirements, or post-processing, which implements pruning mechanisms and rejection of some unsatisfactory rules. The paper focuses on this latter approach.

One of the most straightforward ways to prune rules and rule sets involves exploiting direct parameters of rules, such as their support, length [11], strength [1]. Also specific condition attributes can be taken into account and indicate rules to be selected by appearing in their premises [12]. Such process can lead to improved performance or structure and in the presented research it is compared to weighting of rules by calculated quality measures, also based on attributes [13], both procedures actively using rankings of considered characteristic features [7].

The paper is organised as follows. Section 2 briefly describes some elements of background, that is feature weighting and ranking, and aims of pruning of

(C) The Author(s) 2016

T. Czachórski et al. (Eds.): ISCIS 2016, CCIS 659, pp. 106-114, 2016.

DOI: 10.1007/978-3-319-47217-1_12 
rules and rule sets. Section 3 explains the proposed research framework, details experimental setup, and gives test results. Section 4 concludes the paper.

\section{Background}

The research described in this paper incorporates characteristic feature weights and rankings into the problem of pruning of decision rules and rule sets.

\subsection{Feature Ranking}

Roles of specific features exploited in any classification task can vary in significance and relevance in a high degree. The importance of individual attributes can be discovered by some approach leading to their ranking, that is assigning values of a score function which causes putting them in a specific order [7].

Rankings of characteristic features can be obtained through application of statistical measures, machine learning approaches, or systematic procedures [12]. The former assign calculated weights to all variables, while the latter can return only the positions in a ranking, reflecting discovered order of relevance.

Information Gain coefficient (InfoGain, $I G$ ) is defined by employing the concept of entropy from information theory for attributes and classes:

$$
\operatorname{InfoGain}\left(C l, a_{f}\right)=H(C l)-H\left(C l \mid a_{f}\right),
$$

where $H(C l)$ denotes the entropy for the decision attribute $\mathrm{Cl}$ and $H\left(C l \mid a_{f}\right)$ condition entropy, that is class entropy while observing values of attribute $a$.

An attribute relevance measure can be based on rule length [11], with special attention given to the shortest rules that often possess good generalisation properties:

$$
\operatorname{MREVM}(a)=\operatorname{Nr}(a, \operatorname{MinL}): \operatorname{Nr}(a, \operatorname{MinL}+1),
$$

where $N r(a, L)$ denotes the number of rules with length $L$ in which attribute $a$ appears, and $M i n L$ is the length of the shortest rule containing $a$. The attribute ranking constructed in this way is wrapped around the specific inducer, not its performance, since other parameters of rules are disregarded, but structure.

\subsection{Pruning of Decision Rules}

To limit the number of rules three approaches can be considered [8]:

- pre-processing - the input data is reduced before the learning stage starts by rejecting some examples or cutting down on characteristic features. With less data to infer from, it follows that fewer rules are induced.

- at the algorithm construction stage - by implementation of specific procedures only some rules meeting requirements are found instead of all possible.

- post-processing - the set of inferred rules is analysed and some of its elements discarded while others selected. 
When lower numbers of rules are found the learning stage can be shorter, yet solutions are not necessarily the best. If higher numbers of rules are generated, more thorough and in-depth analysis is enabled, yet even for rule sets with small cardinalities some measures of quality or interestingness can be employed [6].

Rule quality can be weighted by conditional attributes [13]:

$$
Q M\left(r_{i}\right)=\prod_{j=1}^{K_{r_{i}}} w\left(a_{j}\right),
$$

where $K_{r_{i}}$ denotes the number of conditions included in rule $r_{i}$ and $w\left(a_{j}\right)$ weight of $a_{j}$ attribute taken from a ranking. It is assumed that $w\left(a_{j}\right) \in(0,1]$.

\section{Experimental Setup and Obtained Results}

The research works presented were executed within the general framework:

- Initial preparation of learning and testing data sets

- Obtaining rankings of attributes

- Induction of decision algorithms

- Pruning of decision rules in two approaches:

- Selecting rules referring to specific attributes in the ranking

- Calculating measures for all rules while exploiting weights assigned to positions in the attribute rankings, which led to weighting of rules and their rankings, and from these rankings rules in turn were selected

- Comparison and analysis of obtained test results

Steps of these procedures are described in the following subsections.

\subsection{Input Datasets}

As a domain of application for the research stylometric analysis of texts was selected. Stylometry enables authorship attribution while basing on employed linguistic characteristic features. Typically they refer to lexical and syntactic markers, giving frequencies of occurrence for selected function words and punctuation marks that reflect individual habits of sentence and paragraph formation.

Learning and testing samples corresponded to parts of longer works by two pairs of writers, female and male, giving binary classification with balanced data.

As attribute values specified usage frequencies of textual descriptors, they were small fractions, which means that for data mining there was needed either some technique that can deal efficiently with continuous numbers, or some discretization strategy was required [2]. Since regardless of a selected method discretization always causes some loss of information, it was not attempted. 


\subsection{Rankings of Attributes}

In the research presented two attribute rankings were tested. The first one relied on statistical properties detected in input datasets and was completely independent on the classifier used later for prediction, and the other was wrapped around characteristics of induced rules, observing how often each variable occurs in shortest rules, which usually are of higher quality as they are better at generalisation and description of detected patterns than those with many conditions. Orderings of variables for both rankings and both datasets are given in Table 1 .

Table 1. Rankings of condition attributes

\begin{tabular}{|c|c|c|c|c|c|}
\hline \multirow[t]{2}{*}{ No } & \multirow[t]{2}{*}{$w(a)$} & \multicolumn{2}{|c|}{ Female writers } & \multicolumn{2}{|c|}{ Male writers } \\
\hline & & InfoGain & $M R E V M$ & InfoGain & $M R E V M$ \\
\hline 1 & 1 & not & not & and & and \\
\hline 2 & $1 / 2$ & $:$ & $:$ & that & by \\
\hline 3 & $1 / 3$ & ; & but & by & from \\
\hline 4 & $1 / 4$ & , & and & but & of \\
\hline 5 & $1 / 5$ & - & . & from & in \\
\hline 6 & $1 / 6$ & on & , & what & $:$ \\
\hline 7 & $1 / 7$ & $?$ & by & for & $!$ \\
\hline 8 & $1 / 8$ & ( & for & - & on \\
\hline 9 & $1 / 9$ & as & to & $?$ & , \\
\hline 10 & $1 / 10$ & but & this & if & as \\
\hline 11 & $1 / 11$ & by & as & at & ( \\
\hline 12 & $1 / 12$ & that & what & with & with \\
\hline 13 & $1 / 13$ & for & $!$ & not & \\
\hline 14 & $1 / 14$ & to & from & $:$ & this \\
\hline 15 & $1 / 15$ & at & $?$ & to & at \\
\hline 16 & $1 / 16$ &. & - & in & not \\
\hline 17 & $1 / 17$ & and & of & ( & ; \\
\hline 18 & $1 / 18$ & in & in & as & $?$ \\
\hline 19 & $1 / 19$ & this & that & $!$ & - \\
\hline 20 & $1 / 20$ & $!$ & with & ; & to \\
\hline 21 & $1 / 21$ & with & if & on & if \\
\hline 22 & $1 / 22$ & of & at & . & what \\
\hline 23 & $1 / 23$ & what & ( & of & for \\
\hline 24 & $1 / 24$ & if & on & this & but \\
\hline 25 & $1 / 25$ & from & ; & , & that \\
\hline
\end{tabular}


InfoGain returns a specific score for each feature while $M R E V M$ gives a ratio. To unify numbers considered as attribute weights they were assigned in an arbitrary manner, listed in column denoted $w(a)$, and equal $1 / i$, where $i$ is a position in the ranking. Thus the distances between weights decrease while going down the ranking. It is assumed that each variable has nonzero weight.

\subsection{DRSA Rule Classifiers}

The rules were induced with the help of 4eMka Software (developed at the Poznań University of Technology, Poland), which implements Dominance-Based Rough Set Approach (DRSA). By substituting the original indiscernibility relation [4] of classical rough sets with dominance DRSA observes ordinal properties in datasets and enables both nominal and ordinal classification [10].

As the reference points classification systems with all rules on examples were taken. For female writers the algorithm consisted of 62383 rules, which with constraints on minimal rule support to be equal at least 66 resulted in 17 decision rules giving the maximal classification accuracy of $86.67 \%$. For male writers the algorithm contained 46191 rules, limited to 80 by support equal at least 41 , and it gave the correct recognition of $76.67 \%$ of testing samples. In all cases ambiguous decisions were treated as incorrect, without any further processing.

\subsection{Pruning of Rule Sets by Attributes}

Selection of decision rules while following attribute rankings was executed as follows: at $i$-th step only the rules with conditions on the $i$ highest ranking features were taken into account. The rules could refer to all or some proper subsets of variables considered, and these with at least one condition on any of lower ranking attributes were discarded. Thus at the first step only rules with single conditions on the highest ranking variable were filtered, while at the last 25-th step all features and all rules were included. For example at 5-th step for female writer dataset for InfoGain ranking only rules referring to any combination of attributes: not, colon, semicolon, comma, hyphen, were selected. The detailed results for both datasets and both rankings are listed in Table 2.

It can be observed that with each variable added to the studied set the numbers of recalled rules rose significantly, but the classification accuracy equal to or even higher than the reference points was detected quite soon in processing, for InfoGain for female dataset after selection of just four highest ranking attributes, for male writers and $M R E V M$ for just three most important features.

\subsection{Pruning of Rule Sets Through Rule Rankings}

Calculation of $Q M$ measure for rules can be understood as translating feature rankings into rule rankings. Depending on cardinalities of subsets of rules selected at each step, the total number of executed steps can significantly vary. The minimum is obviously one, while the maximum can even equal the total number of rules in the analysed set, if with each step only a single rule is added. 
Table 2. Characteristics of decision algorithms with pruning of rules referring to specific conditional attributes: $\mathrm{N}$ indicates the number of considered attributes, (a) number of recalled rules, (b) maximal classification accuracy [\%], (c) minimal support required of rules, (d) number of rules satisfying condition on support

\begin{tabular}{|c|c|c|c|c|c|c|c|c|c|c|c|c|c|c|c|c|}
\hline \multirow[t]{3}{*}{$\mathrm{N}$} & \multicolumn{8}{|c|}{ Female } & \multicolumn{8}{|l|}{ Male } \\
\hline & \multicolumn{4}{|c|}{ InfoGain } & \multicolumn{4}{|c|}{$M R E V M$} & \multicolumn{4}{|c|}{ Info Gain } & \multicolumn{4}{|c|}{$M R E V M$} \\
\hline & (a) & (b) & (c) & (d) & (a) & (b) & (c) & (d) & (a) & (b) & (c) & (d) & (a) & (b) & (c) & (d) \\
\hline 1 & 10 & 61.11 & 55 & 4 & 10 & 61.11 & 55 & 10 & 6 & 13.33 & 14 & 4 & 6 & 13.33 & 14 & 4 \\
\hline 2 & 27 & 81.11 & 55 & 13 & 27 & 81.11 & 55 & 13 & 15 & 21.11 & 9 & 8 & 27 & 55.56 & 9 & 23 \\
\hline 3 & 36 & 81.11 & 55 & 13 & 56 & 82.22 & 32 & 27 & 45 & 61.11 & 6 & 35 & 80 & 80.00 & 25 & 39 \\
\hline 4 & 79 & 86.67 & 55 & 27 & 97 & 81.11 & 55 & 14 & 73 & 61.11 & 10 & 41 & 127 & 80.00 & 25 & 50 \\
\hline 5 & 91 & 86.67 & 55 & 27 & 203 & 82.22 & 44 & 26 & 153 & 75.56 & 21 & 62 & 219 & 81.11 & 20 & 115 \\
\hline 6 & 128 & 86.67 & 55 & 27 & 324 & 86.67 & 55 & 28 & 198 & 75.56 & 21 & 65 & 290 & 80.00 & 41 & 25 \\
\hline 7 & 167 & 86.67 & 55 & 27 & 578 & 86.67 & 55 & 30 & 239 & 75.56 & 26 & 46 & 562 & 75.56 & 41 & 28 \\
\hline 8 & 202 & 86.67 & 55 & 27 & 877 & 86.67 & 66 & 13 & 307 & 75.56 & 21 & 72 & 778 & 75.56 & 41 & 29 \\
\hline 9 & 356 & 86.67 & 66 & 11 & 1317 & 86.67 & 66 & 13 & 422 & 75.56 & 21 & 79 & 1073 & 75.56 & 41 & 30 \\
\hline 10 & 570 & 86.67 & 66 & 11 & 1923 & 86.67 & 66 & 15 & 531 & 75.56 & 21 & 89 & 1355 & 75.56 & 41 & 31 \\
\hline 11 & 1011 & 86.67 & 66 & 12 & 2755 & 86.67 & 66 & 16 & 689 & 75.56 & 32 & 44 & 1591 & 78.89 & 41 & 41 \\
\hline 12 & 1415 & 86.67 & 66 & 14 & 3793 & 86.67 & 66 & 16 & 866 & 75.56 & 32 & 48 & 1975 & 76.67 & 41 & 45 \\
\hline 13 & 2201 & 86.67 & 66 & 14 & 4995 & 86.67 & 66 & 17 & 1395 & 75.56 & 32 & 65 & 3169 & 76.67 & 41 & 45 \\
\hline 14 & 3137 & 86.67 & 66 & 14 & 6671 & 86.67 & 66 & 17 & 1763 & 75.56 & 32 & 67 & 4456 & 78.89 & 41 & 53 \\
\hline 15 & 4215 & 86.67 & 66 & 14 & 8099 & 86.67 & 66 & 17 & 2469 & 75.56 & 32 & 67 & 5774 & 78.89 & 41 & 53 \\
\hline 16 & 5473 & 86.67 & 66 & 14 & 9485 & 86.67 & 66 & 17 & 3744 & 75.56 & 41 & 42 & 8476 & 76.67 & 41 & 63 \\
\hline 17 & 7901 & 86.67 & 66 & 14 & 13255 & 86.67 & 66 & 17 & 4336 & 75.56 & 41 & 56 & 11055 & 76.67 & 41 & 66 \\
\hline 18 & 10732 & 86.67 & 66 & 14 & 17589 & 86.67 & 66 & 17 & 5352 & 75.56 & 41 & 57 & 13428 & 76.67 & 41 & 69 \\
\hline 19 & 14187 & 86.67 & 66 & 16 & 21238 & 86.67 & 66 & 17 & 7214 & 75.56 & 41 & 60 & 16188 & 76.67 & 41 & 69 \\
\hline 20 & 18087 & 86.67 & 66 & 17 & 26821 & 86.67 & 66 & 17 & 9819 & 75.56 & 41 & 63 & 22035 & 76.67 & 41 & 75 \\
\hline 21 & 23408 & 86.67 & 66 & 17 & 33834 & 86.67 & 66 & 17 & 14282 & 75.56 & 41 & 64 & 26674 & 76.67 & 41 & 78 \\
\hline 22 & 31050 & 86.67 & 66 & 17 & 43225 & 86.67 & 66 & 17 & 18590 & 75.56 & 41 & 64 & 30846 & 76.67 & 41 & 78 \\
\hline 23 & 39235 & 86.67 & 66 & 17 & 52587 & 86.67 & 66 & 17 & 26474 & 75.56 & 41 & 70 & 36630 & 76.67 & 41 & 78 \\
\hline 24 & 48583 & 86.67 & 66 & 17 & 58097 & 86.67 & 66 & 17 & 35014 & 76.67 & 41 & 79 & 40024 & 76.67 & 41 & 78 \\
\hline 25 & 62383 & 86.67 & 66 & 17 & & & & & 46191 & 76.67 & 41 & 80 & & & & \\
\hline
\end{tabular}

On the other hand, once the core sets of rules, corresponding to the decision algorithms limited by constraints on minimal support of rules and giving the best results for the complete algorithms, are retrieved, there is little point in continuing, thus the results presented in Table 3 stop when only fractions of the whole rule sets are recalled, for female writers just few hundreds, and for male writers close to ten thousand (still less than a quarter of the original algorithm).

\subsection{Summary of the Best Results}

Out of the two tested and compared approaches to rule filtering, selection governed by attributes included when following their rankings enabled to reject more rules from the reference algorithms, even over $35 \%$ and $48 \%$, respectively for female and male datasets, with prediction at the reference level. For male writers recognition could be increased (at maximum by over $4 \%$ ) either with keeping or lowering constraints on minimal support required of rules. 
Table 3. Characteristics of decision algorithms with pruning of rules while weighting them by measures based on rankings of conditional attributes: $\mathrm{N}$ indicates the weighting step, (a) number of recalled rules, (b) maximal classification accuracy [\%], (c) minimal support required of rules, (d) number of rules satisfying condition on support

\begin{tabular}{|c|c|c|c|c|c|c|c|c|c|c|c|c|c|c|c|c|}
\hline \multirow[t]{3}{*}{$\mathrm{N}$} & \multicolumn{8}{|c|}{ Female } & \multicolumn{8}{|l|}{ Male } \\
\hline & \multicolumn{4}{|c|}{ InfoGain-RDD } & \multicolumn{4}{|c|}{$M R E V M-\mathrm{RDD}$} & \multicolumn{4}{|c|}{ InfoGain-RDD } & \multicolumn{4}{|c|}{$M R E V M-\mathrm{RDD}$} \\
\hline & (a) & (b) & (c) & (d) & (a) & (b) & (c) & (d) & (a) & (b) & (c) & (d) & (a) & (b) & (c) & (d) \\
\hline 1 & 10 & 61.11 & 55 & 4 & 10 & 61.11 & 55 & 4 & 36 & 55.56 & 9 & 26 & 27 & 55.56 & 9 & 23 \\
\hline 2 & 12 & 61.11 & 55 & 4 & 12 & 61.11 & 55 & 4 & 113 & 61.11 & 13 & 58 & 48 & 61.11 & 13 & 39 \\
\hline 3 & 29 & 81.11 & 55 & 13 & 39 & 83.33 & 32 & 23 & 128 & 61.11 & 13 & 62 & 60 & 61.11 & 13 & 45 \\
\hline 4 & 46 & 87.78 & 52 & 25 & 55 & 84.44 & 14 & 37 & 154 & 61.11 & 13 & 70 & 71 & 61.11 & 13 & 53 \\
\hline 5 & 48 & 87.78 & 52 & 25 & 70 & 84.44 & 14 & 45 & 185 & 66.67 & 10 & 99 & 112 & 80.00 & 25 & 52 \\
\hline 6 & 67 & 87.78 & 52 & 25 & 104 & 87.78 & 52 & 28 & 215 & 66.67 & 10 & 120 & 127 & 73.33 & 26 & 56 \\
\hline 7 & 71 & 87.78 & 52 & 25 & 129 & 87.78 & 52 & 31 & 231 & 66.67 & 10 & 130 & 149 & 73.33 & 26 & 63 \\
\hline 8 & 80 & 90.00 & 46 & 29 & 161 & 87.78 & 52 & 36 & 265 & 73.33 & 26 & 86 & 189 & 73.33 & 26 & 66 \\
\hline 9 & 94 & 90.00 & 46 & 33 & 182 & 87.78 & 52 & 39 & 301 & 73.33 & 26 & 90 & 251 & 73.33 & 26 & 79 \\
\hline 10 & 106 & 90.00 & 46 & 33 & 212 & 88.89 & 52 & 45 & 329 & 73.33 & 26 & 99 & 288 & 73.33 & 26 & 87 \\
\hline 11 & 131 & 90.00 & 46 & 38 & 226 & 88.89 & 52 & 48 & 384 & 73.33 & 26 & 110 & 331 & 73.33 & 41 & 33 \\
\hline 12 & 166 & 86.67 & 66 & 12 & 265 & 86.67 & 66 & 16 & 396 & 73.33 & 26 & 116 & 368 & 73.33 & 41 & 41 \\
\hline 13 & 181 & 86.67 & 66 & 14 & 279 & 86.67 & 66 & 17 & 511 & 73.33 & 26 & 124 & 382 & 73.33 & 41 & 44 \\
\hline 14 & 202 & 86.67 & 66 & 14 & 327 & 86.67 & 66 & 17 & 667 & 75.56 & 25 & 143 & 451 & 73.33 & 41 & 48 \\
\hline 15 & 206 & 86.67 & 66 & 14 & 339 & 86.67 & 66 & 17 & 794 & 75.56 & 32 & 91 & 483 & 75.56 & 27 & 130 \\
\hline 16 & 221 & 86.67 & 66 & 14 & 362 & 86.67 & 66 & 17 & 912 & 73.33 & 32 & 94 & 514 & 76.67 & 27 & 135 \\
\hline 17 & 237 & 86.67 & 66 & 14 & 388 & 86.67 & 66 & 17 & 949 & 73.33 & 26 & 148 & 624 & 75.56 & 37 & 74 \\
\hline 18 & 268 & 86.67 & 66 & 14 & 441 & 86.67 & 66 & 17 & 1011 & 73.33 & 41 & 54 & 848 & 75.56 & 37 & 77 \\
\hline 19 & 285 & 86.67 & 66 & 16 & 452 & 86.67 & 66 & 17 & 1117 & 75.56 & 27 & 153 & 937 & 78.89 & 35 & 87 \\
\hline 20 & 305 & 86.67 & 66 & 17 & 498 & 86.67 & 66 & 17 & 1189 & 75.56 & 27 & 155 & 1236 & 76.67 & 35 & 91 \\
\hline 21 & & & & & & & & & 1228 & 75.56 & 27 & 157 & 1965 & 76.67 & 41 & 65 \\
\hline 22 & & & & & & & & & 1900 & 75.56 & 41 & 61 & 2160 & 76.67 & 41 & 67 \\
\hline 23 & & & & & & & & & 1993 & 75.56 & 41 & 63 & 2264 & 76.67 & 41 & 68 \\
\hline 24 & & & & & & & & & 2667 & 76.67 & 41 & 67 & 3291 & 76.67 & 41 & 71 \\
\hline 25 & & & & & & & & & 3610 & 76.67 & 41 & 68 & 4036 & 76.67 & 41 & 72 \\
\hline 26 & & & & & & & & & 4577 & 76.67 & 41 & 70 & 4519 & 76.67 & 41 & 74 \\
\hline 27 & & & & & & & & & 4825 & 76.67 & 41 & 71 & 5637 & 76.67 & 41 & 76 \\
\hline 28 & & & & & & & & & 5725 & 76.67 & 41 & 74 & 6269 & 76.67 & 41 & 77 \\
\hline 29 & & & & & & & & & 7901 & 76.67 & 41 & 76 & 9820 & 76.67 & 41 & 79 \\
\hline 30 & & & & & & & & & 9250 & 76.67 & 41 & 78 & 9830 & 76.67 & 41 & 80 \\
\hline 31 & & & & & & & & & 9394 & 76.67 & 41 & 79 & 9841 & 76.67 & 41 & 80 \\
\hline 32 & & & & & & & & & 9404 & 76.67 & 41 & 80 & 9844 & 76.67 & 41 & 80 \\
\hline
\end{tabular}

When rules were wighted, ranked, and then selected the quality of prediction was enhanced at maximum by over $3 \%$ for both datasets, and for female and male writers datasets respectively over $29 \%$ and $18 \%$ of rules could be pruned.

For female dataset for both approaches to rule pruning better results were obtained while exploiting InfoGain attribute ranking, and for male dataset the same can be stated for MREVM ranking. 


\section{Conclusions}

The paper presents research on selection of decision rules while following rankings of considered conditional attributes and exploiting weights assigned to them, which constitute alternatives to the popular approaches to rule filtering. Two ways to prune rules were compared, the first relying on selection of the rules with conditions only on the highest ranking attributes, while those referring to lower ranking features were rejected. Within the second methodology, the weights of attributes from their rankings formed a base from which for all rules the defined quality measures were calculated, and their values led to rule rankings. Next, the highest ranking rules were filtered out. For both described approaches two attribute rankings were tested, and the test results show several possibilities of constructing optimised rule classifiers, either with increased recognition, decreased lengths of decision algorithms, or both.

Acknowledgments. The research presented was performed at the Silesian University of Technology, Gliwice, Poland, within the project BK/RAu2/2016.

Open Access. This chapter is distributed under the terms of the Creative Commons Attribution 4.0 International License (http://creativecommons.org/licenses/by/ $4.0 /$ ), which permits use, duplication, adaptation, distribution and reproduction in any medium or format, as long as you give appropriate credit to the original author(s) and the source, a link is provided to the Creative Commons license and any changes made are indicated.

The images or other third party material in this chapter are included in the work's Creative Commons license, unless indicated otherwise in the credit line; if such material is not included in the work's Creative Commons license and the respective action is not permitted by statutory regulation, users will need to obtain permission from the license holder to duplicate, adapt or reproduce the material.

\section{References}

1. Amin, T., Chikalov, I., Moshkov, M., Zielosko, B.: Relationships between length and coverage of decision rules. Fundamenta Informaticae 129, 1-13 (2014)

2. Baron, G.: On approaches to discretization of datasets used for evaluation of decision systems. In: Czarnowski, I., Caballero, A., Howlett, R., Jain, L. (eds.) Intelligent Decision Technologies 2016. Smart Innovation, Systems and Technologies, vol. 56, pp. 149-159. Springer, Switzerland (2016)

3. Bayardo Jr., R., Agrawal, R.: Mining the most interesting rules. In: Proceedings of the 5th ACM SIGKDD International Conference on Knowledge Discovery and Data Mining, pp. 145-154 (1999)

4. Cyran, K.A., Stanczyk, U.: Indiscernibility relation for continuous attributes: application in image recognition. In: Kryszkiewicz, M., Peters, J.F., Rybiński, H., Skowron, A. (eds.) RSEISP 2007. LNCS (LNAI), vol. 4585, pp. 726-735. Springer, Heidelberg (2007)

5. Fürnkranz, J., Gamberger, D., Lavrač, N.: Foundations of Rule Learning. Springer, Heidelberg (2012) 
6. Gruca, A., Sikora, M.: Rule based functional description of genes - estimation of the multicriteria rule interestingness measure by the UTA method. Biocybernetics Biomed. Eng. 33, 222-234 (2013)

7. Mansoori, E.: Using statistical measures for feature ranking. Int. J. Pattern Recog. Artitf. Intell. 27(1), 1350003-1350014 (2013)

8. Sikora, M.: Induction and pruning of classification rules for prediction of microseismic hazards in coal mines. Expert Syst. Appl. 38(2), 6748-6758 (2013)

9. Sikora, M., Wróbel, Ł.: Data-driven adaptive selection of rules quality measures for improving the rules induction algorithm. In: Kuznetsov, S.O., Ślęzak, D., Hepting, D.H., Mirkin, B.G. (eds.) RSFDGrC 2011. LNCS (LNAI), vol. 6743, pp. 278-285. Springer, Heidelberg (2011). doi:10.1007/978-3-642-21881-1_44

10. Słowiński, R., Greco, S., Matarazzo, B.: Dominance-based rough set approach to reasoning about ordinal data. In: Kryszkiewicz, M., Peters, J.F., Rybinski, H., Skowron, A. (eds.) RSEISP 2007. LNCS (LNAI), vol. 4585, pp. 5-11. Springer, Heidelberg (2007). doi:10.1007/978-3-540-73451-2_2

11. Stańczyk, U.: Decision rule length as a basis for evaluation of attribute relevance. J. Intell. Fuzzy Syst. 24(3), 429-445 (2013)

12. Stańczyk, U.: Selection of decision rules based on attribute ranking. J. Intell. Fuzzy Syst. 29(2), 899-915 (2015)

13. Stańczyk, U.: Measuring quality of decision rules through ranking of conditional attributes. In: Czarnowski, I., Caballero, A., Howlett, R., Jain, L. (eds.) Intelligent Decision Technologies 2016. Smart Innovation, Systems and Technologies, vol. 56, pp. 269-279. Springer, Switzerland (2016) 